difficult, and probably not possible, to express $\mathbf{f}$ as the sum of a finite number of terms for each of which we can use (3).

\title{
STRESS FUNCTIONS OF MAXWELL AND MORERA*
}

\section{Br WILHELM ORNSTEIN (Newark College of Engineering)}

Summary. A systematic process is devised for the derivation of the stress functions of Maxwell and Morera by the application of the theorem that a vector whose divergence vanishes is solenoidal. Symmetric and anti-symmetric matrices are established, and the elements of these matrices represent the stress functions of Maxwell and Morera respectively.

Introduction. The procedure used by $\mathrm{Maxwell}^{1}$ to derive three stress functions representing six stress components at any point of an isotropic body is similar to that which Morera ${ }^{2}$ subsequently applied to the establishment of the corresponding stress functions. This procedure described also by Love ${ }^{3}$ consisted in the choice of three stress components; then the substitution of these components into the equilibrium equation led to the remaining three components necessary to satisfy the equations of equilibrium. Later it was discovered by Sir Richard Southwell ${ }^{4}$ that Saint Venant's and Beltrami's compatibility equations follow from Castigliano's principle when the strain energy is expressed in terms of Maxwell's and of Morera's functions.

Derivations. Neglecting the body forces, the equation of equilibrium is

$$
\operatorname{div} T=0 \text {, }
$$

where $T$ is the stress tensor. The three equations of equilibrium are obtained by cyclic interchange of $x, y, z$ in the equation

$$
\frac{\partial X x}{\partial x}+\frac{\partial X y}{\partial y}+\frac{\partial X z}{\partial z}=0 \text {. }
$$

The equations of equilibrium (2) can be written as

$$
\operatorname{div} A=0, \quad \operatorname{div} B=0, \quad \operatorname{div} C=0
$$

where, because of the theorem that a vector whose divergence vanishes is a solenoidal vector, the following relations exist:

$$
A=\operatorname{curl} F, \quad B=\operatorname{curl} G, \quad C=\operatorname{curl} H .
$$

Consequently, the stress components may be written as follows:

$$
X x=\frac{\partial F_{3}}{\partial y}-\frac{\partial F_{2}}{\partial z}, \quad X y=\frac{\partial F_{1}}{\partial z}-\frac{\partial F_{3}}{\partial x}, \quad X z=\frac{\partial F_{2}}{\partial x}-\frac{\partial F_{1}}{\partial y} ;
$$

*Received September 2, 1953.

${ }^{1}$ C. Maxwell, Edinburgh Royal Soc. Trans. 26 (1870).

${ }^{2}$ G. Morera, Roma, Acc. Lincei Rend. (5) 1 (1892).

${ }^{3}$ A. E. H. Love, $A$ treatise on the mathematical theory of elasticity, Fourth Edition, 1927, p. 88.

${ }^{4}$ R. V. Southwell, Proc. Roy. Soc. (A) 154 (1925). R. V. Southwell, Timoshenko Anniversary Volume, pp. 211-216. 


$$
\begin{array}{lll}
Y x=\frac{\partial G_{3}}{\partial y}-\frac{\partial G_{2}}{\partial z}, & Y y=\frac{\partial G_{1}}{\partial z}-\frac{\partial G_{3}}{\partial x}, & Y z=\frac{\partial G_{2}}{\partial x}-\frac{\partial G_{1}}{\partial y} \\
Z x=\frac{\partial H_{3}}{\partial y}-\frac{\partial H_{2}}{\partial z}, & Z y=\frac{\partial H_{1}}{\partial z}-\frac{\partial H_{3}}{\partial x}, & Z z=\frac{\partial H_{2}}{\partial x}-\frac{\partial H_{1}}{\partial y} .
\end{array}
$$

Upon substituting (5) into the equilibrium conditions

$$
X y=Y x ; \quad X z=Z x ; \quad Y z=Z y
$$

and introducing, for abbreviation, the symbol $\Gamma=F_{1}+G_{2}+H_{3}$, the following equations are obtained:

$$
\begin{aligned}
& \frac{\partial F_{3}}{\partial x}+\frac{\partial G_{3}}{\partial y}+\frac{\partial\left(H_{3}-\Gamma\right)}{\partial z}=0 \\
& \frac{\partial F_{2}}{\partial x}+\frac{\partial\left(G_{2}-\Gamma\right)}{\partial y}+\frac{\partial H_{2}}{\partial z}=0 \\
& \frac{\partial\left(F_{1}-\Gamma\right)}{\partial x}+\frac{\partial G_{1}}{\partial y}+\frac{\partial H_{1}}{\partial z}=0 .
\end{aligned}
$$

Equations (7), in the same manner as Eqs. (2), can be expressed as before: when the divergence of some vector is equal to zero, that vector is solenoidal. Thus the following expressions can be written:

$$
\begin{aligned}
& F_{1}-\Gamma=\frac{\partial U_{3}}{\partial y}-\frac{\partial U_{2}}{\partial z}, \\
& G_{1}=\frac{\partial U_{1}}{\partial z}-\frac{\partial U_{3}}{\partial x}, \\
& H_{1}=\frac{\partial U_{2}}{\partial x}-\frac{\partial U_{1}}{\partial y} \\
& F_{2}=\frac{\partial V_{3}}{\partial y}-\frac{\partial V_{2}}{\partial z}, \quad G_{2}-\Gamma=\frac{\partial V_{1}}{\partial z}-\frac{\partial V_{3}}{\partial x}, \\
& H_{2}=\frac{\partial V_{2}}{\partial x}-\frac{\partial V_{1}}{\partial y} \\
& F_{3}=\frac{\partial W_{3}}{\partial y}-\frac{\partial W_{2}}{\partial z} \\
& G_{3}=\frac{\partial W_{1}}{\partial z}-\frac{\partial W_{3}}{\partial x} \\
& H_{3}-\Gamma=\frac{\partial W_{2}}{\partial x}-\frac{\partial W_{1}}{\partial y} .
\end{aligned}
$$

To evaluate the expression $\Gamma$, the three terms on the diagonal of Eqs. (8) are added:

from which

$$
F_{1}+G_{2}+H_{3}-3 \Gamma=-2 \Gamma
$$

$$
\Gamma=-\frac{1}{2} \frac{\partial}{\partial x}\left(W_{2}-V_{3}\right)-\frac{1}{2} \frac{\partial}{\partial y}\left(U_{3}-W_{1}\right)-\frac{1}{2} \frac{\partial}{\partial z}\left(V_{1}-U_{2}\right) .
$$

Upon substitution of (9) into (8) the following relations are obtained:

$$
\begin{aligned}
& F_{1}=\frac{\partial U_{3}}{\partial y}-\frac{\partial U_{2}}{\partial z}-\frac{1}{2} \frac{\partial}{\partial x}\left(W_{2}-V_{3}\right)-\frac{1}{2} \frac{\partial}{\partial y}\left(U_{3}-W_{1}\right)-\frac{1}{2} \frac{\partial}{\partial z}\left(V_{1}-U_{2}\right), \\
& G_{2}=\frac{\partial V_{1}}{\partial z}-\frac{\partial V_{3}}{\partial x}-\frac{1}{2} \frac{\partial}{\partial x}\left(W_{2}-V_{3}\right)-\frac{1}{2} \frac{\partial}{\partial y}\left(U_{3}-W_{1}\right)-\frac{1}{2} \frac{\partial}{\partial z}\left(V_{1}-U_{2}\right), \\
& H_{3}=\frac{\partial W_{2}}{\partial x}-\frac{\partial W_{1}}{\partial y}-\frac{1}{2} \frac{\partial}{\partial x}\left(W_{2}-V_{3}\right)-\frac{1}{2} \frac{\partial}{\partial y}\left(U_{3}-W_{1}\right)-\frac{1}{2} \frac{\partial}{\partial z}\left(V_{1}-U_{2}\right)
\end{aligned}
$$

Now, by use of (8) and (10) the stresses in (5) can be determined as follows:

$$
X x=\frac{\partial^{2} W_{3}}{\partial y^{2}}+\frac{\partial^{2} V_{2}}{\partial z^{2}}-\frac{\partial^{2}}{\partial y \partial z}\left(W_{2}+V_{3}\right),
$$




$$
\begin{aligned}
Y y & =\frac{\partial^{2} U_{1}}{\partial z^{2}}+\frac{\partial^{2} W_{3}}{\partial x^{2}}-\frac{\partial^{2}}{\partial x \partial z}\left(U_{3}+W_{1}\right) \\
Z z & =\frac{\partial^{2} V_{2}}{\partial x^{2}}+\frac{\partial^{2} U_{1}}{\partial y^{2}}-\frac{\partial^{2}}{\partial x \partial y}\left(V_{1}+U_{2}\right) \\
X y & =\frac{1}{2} \frac{\partial^{2} U_{3}}{\partial y \partial z}-\frac{1}{2} \frac{\partial^{2} U_{2}}{\partial z^{2}}-\frac{\partial^{2} W_{3}}{\partial x \partial y}+\frac{1}{2} \frac{\partial^{2} W_{2}}{\partial x \partial z}+\frac{1}{2} \frac{\partial^{2} V_{3}}{\partial z \partial x}+\frac{1}{2} \frac{\partial^{2} W_{1}}{\partial y \partial}-\frac{1}{2} \frac{\partial^{2} V_{1}}{\partial z^{2}} \\
& =\frac{1}{2} \frac{\partial}{\partial z}\left\{-\frac{\partial}{\partial z}\left(V_{1}+U_{2}\right)+\frac{\partial}{\partial x}\left(W_{2}+V_{3}\right)+\frac{\partial}{\partial y}\left(U_{3}+W_{1}\right)\right\}-\frac{\partial^{2} W_{3}}{\partial x \partial y} \\
X y & =Y x=-\frac{1}{2} \frac{\partial^{2}\left(U_{2}+V_{1}\right)}{\partial z^{2}}+\frac{1}{2} \frac{\partial^{2}\left(W_{2}+V_{3}\right)}{\partial x \partial z}+\frac{1}{2} \frac{\partial^{2}\left(U_{3}+W_{1}\right)}{\partial y \partial z}-\frac{\partial^{2} W_{3}}{\partial x \partial y}
\end{aligned}
$$

In a similar way one obtains

$$
\begin{aligned}
& X z=Z x=-\frac{1}{2} \frac{\partial^{2}\left(W_{1}+U_{3}\right)}{\partial y^{2}}+\frac{1}{2} \frac{\partial^{2}\left(W_{2}+V_{3}\right)}{\partial y \partial z}+\frac{1}{2} \frac{\partial^{2}\left(V_{1}+U_{2}\right)}{\partial x \partial y}-\frac{\partial^{2} V_{2}}{\partial x \partial z}, \\
& Y z=Z y=-\frac{1}{2} \frac{\partial^{2}\left(W_{2}+V_{3}\right)}{\partial x^{2}}+\frac{1}{2} \frac{\partial^{2}\left(U_{3}+W_{1}\right)}{\partial x \partial y}+\frac{1}{2} \frac{\partial^{2}\left(V_{1}+U_{2}\right)}{\partial x \partial z}-\frac{\partial^{2} W_{1}}{\partial y \partial z}
\end{aligned}
$$

The normal and tangential stress components expressed by Eqs. (11) to (16) are determined by the elements of a square matrix, which can be written as follows:

$$
\left|\begin{array}{ccc}
U_{1} & U_{2} & U_{3} \\
V_{1} & V_{2} & V_{3} \\
W_{1} & W_{2} & W_{3}
\end{array}\right|
$$

when all the elements except those on the diagonal are set equal to zero; i.e., when

$$
U_{2}=U_{3}=V_{1}=V_{3}=W_{1}=W_{2}=0
$$

and when

$$
U_{1}=\chi_{1}, \quad V_{2}=\chi_{2}, \quad W_{3}=\chi_{3}
$$

there is a symmetric, or diagonal, matrix, whose elements form the Maxwell stress functions. With these elements the stress components are obtained from equations (11) to (16) as follows:

$$
\begin{array}{cc}
X x=\frac{\partial^{2} \chi_{3}}{\partial y^{2}}+\frac{\partial^{2} \chi_{2}}{\partial z^{2}}, & Y y=\frac{\partial^{2} \chi_{1}}{\partial z^{2}}+\frac{\partial^{2} \chi_{3}}{\partial x^{2}}, \\
Z z=\frac{\partial^{2} \chi_{2}}{\partial x^{2}}+\frac{\partial^{2} \chi_{1}}{\partial y^{2}}, & X y=Y x=-\frac{\partial^{2} \chi_{3}}{\partial x \partial y}, \\
Y z=Z y=-\frac{\partial^{2} \chi_{1}}{\partial y \partial z}, & X z=Z x=-\frac{\partial^{2} \chi_{2}}{\partial z \partial x} .
\end{array}
$$

When the diagonal elements of the Matrix (17) are set equal to zero; i.e., when

$$
U_{1}=V_{2}=W_{3}=0
$$


and when

$$
\begin{aligned}
& W_{2}+V_{3}=-\psi_{1} \\
& U_{3}+W_{1}=-\psi_{2} \\
& V_{1}+U_{2}=-\psi_{3}
\end{aligned}
$$

the matrix is anti-symmetric, and its elements represent the Morera stress-functions which when substituted into Eqs. (11) to (16) yield the following stress components:

$$
\begin{aligned}
& X x=\frac{\partial^{2} \psi_{1}}{\partial y \partial z}, \quad Y y=\frac{\partial^{2} \psi_{2}}{\partial z \partial x}, \quad Z z=\frac{\partial^{2} \psi_{3}}{\partial x \partial y} \\
& X y=Y x=-\frac{1}{2} \frac{\partial}{\partial z}\left(\frac{\partial \psi_{1}}{\partial x}+\frac{\partial \psi_{2}}{\partial y}-\frac{\partial \psi_{3}}{\partial z}\right) \\
& X z=Z x=-\frac{1}{2} \frac{\partial}{\partial y}\left(\frac{\partial \psi_{1}}{\partial x}-\frac{\partial \psi_{2}}{\partial y}+\frac{\partial \psi_{3}}{\partial z}\right) \\
& Y z=Z y=-\frac{1}{2} \frac{\partial}{\partial z}\left(-\frac{\partial \psi_{1}}{\partial x}+\frac{\partial \psi_{2}}{\partial y}+\frac{\partial \psi_{3}}{\partial z}\right)
\end{aligned}
$$

Thus, the stress tensor expressed by Maxwell and Morera functions was derived by a direct method from diagonal and antisymmetric matrices.

\section{A REMARK ON INTEGRAL INVARIANTS*}

By H. D. BLOCK (University of Minnesota)

Let the $2 n$ variables $q_{1}, q_{2}, \cdots, q_{n}, p_{1}, p_{2}, \cdots, p_{n}$ be related to the $2 n$ variables $Q_{1}, Q_{2}, \cdots, Q_{n}, P_{1}, P_{2}, \cdots, P_{n}$ by a canonical transformation. Let $\sigma$ be the unit square: $0 \leqq u \leqq 1,0 \leqq v \leqq 1$, and let $q_{i}=f_{i}(u, v), p_{i}=g_{i}(u, v),(i=1,2, \cdots, n)$, where $f_{i}$ and $g_{i}$ have continuous derivatives on $\sigma$. This induces the relationships $Q_{i}=F_{i}$ $(u, v), P_{i}=G_{i}(u, v),(i=1,2, \cdots, n)$. Let $s_{i}=\bigcup_{(u, v) \varepsilon \sigma}\left(f_{i}(u, v), g_{i}(u, v)\right)$ and $S_{i}=$ $\bigcup_{(u, v)=\sigma}\left(F_{i}(u, v), G_{i}(u, v)\right)$, i.e. the maps of $\sigma$ on the $\left(q_{i}, p_{i}\right)$ and $\left(Q_{i}, P_{i}\right)$ planes respectively. Let

$$
\sum_{i=1}^{n} \iint_{s i} d q_{i} d p_{i} \quad \text { and } \quad \sum_{i=1}^{n} \iint_{S_{i}} d Q_{i} d P_{i}
$$

be denoted respectively by

$$
\iint_{i} \sum d q_{i} d p_{i} \quad \text { and } \quad \iint_{S} \sum d Q_{i} d P_{i} .
$$

It is widely ${ }^{1}$ believed that under the conditions stated

$$
\iint_{\cdot} \sum d q_{i} d p_{i}=\iint_{S} \sum d Q_{i} d P_{i} .
$$

*Received Oct. 28, 1953.

${ }^{1}$ Cf. e.g. Goldstein Classical Mechanics, Addison Wesley, 1950, pp. 247-250. Also Corben and Stehle Classical Mechanics, Wiley, 1950, pp. 292-3. 